\title{
AN EXPLANATION FOR THE SHAPE OF NANOINDENTATION UNLOADING CURVES BASED ON FINITE ELEMENT SIMULATION
}

\author{
A. BOLSHAKOV*, W.C. OLIVER**, and G.M. PHARR* \\ *Department of Materials Science, Rice University, P.O. Box 1892, Houston, TX 77251 \\ **Nano Instruments, Inc., P.O. Box 14211, Knoxville, TN 37914
}

\begin{abstract}
Current methods for measuring hardness and modulus from nanoindentation loaddisplacement data are based on Sneddon's equations for the indentation of an elastic half-space by an axially symmetric rigid punch. Recent experiments have shown that nanoindentation unloading data are distinctly curved in a manner which is not consistent with either the flat punch or the conical indenter geometries frequently used in modeling, but are more c approximated by a parabola of revolution. Finite element simulations for conical indentation of an elastic-plastic material are presented which corroborate the experimental observations, and from which a simple explanation for the shape of the unloading curve is derived. The explanation is based on the concept of an effective indenter shape whose geometry is determined by the shape of the plastic hardness impression formed during indentation.
\end{abstract}

\section{'INTRODUCTION}

This paper deals with the shapes of unloading curves obtained in nanoindentation experiments, Analyses of these curves based on Sneddon's equations for the indentation of an elastic half-space by an axisymmetric indenter [1] are frequently used to determine the hardness and elastic modulus of thin film and monolithic materials.

Two different approaches for the analysis of the unloading data have been developed, both of which assume that the material behavior during unloading is completely defined by its elastic properties. In 1986, Doerner and Nix [2] suggested that linear fitting of the unloading data may be used to determine the unloading stiffness, from which the hardness and modulus can be derived. Their approach was justified by experimental observations that the initial portions of unloading curves are approximately linear, as would be expected for the flat-punch indenter geometry. However, a subsequent investigation by Oliver and Pharr [3], which led to a second method for data analysis, showed that none of a wide variety of materials including fused silica, soda-lime glass, and single crystals of aluminum, tungsten, quartz, and sapphire exhibit truly linear unloading. Instead, it was found that unloading curves are better described by the powerlaw relation :

$$
P=A\left(h-h_{f}\right)^{m}
$$

where $\mathrm{P}$ is load, $\mathrm{h}$ is displacement, $\mathrm{h}_{\mathrm{f}}$ is the final displacement after complete unloading, and $\mathrm{A}$ and $m$ are power-law parameters. The values of $A, m$ and $h_{f}$ were determined from regression analysis of the experimental data.

An important observation in the work of Oliver and Pharr is that the values of the power law exponent, while slightly material dependent, generally fall in the range $1.25-1.51$ (1.25 for fused silica; 1.51 for tungsten). As such, observed experimental behavior is consistent with neither the flat punch indenter geometry $(m=1)$, nor the conical indenter $(m=2)$. The disagreement with the latter geometry is particularly disconcerting given that a cone most closely approximates the self-similar geometry of the Berkovich indenter used to obtain the experimental data. Curiously, an indenter with the geometry of a parabola of revolution, for which $\mathrm{m}=1.5$, comes closest to predicting the experimental observations. However, how a sharp Berkovich
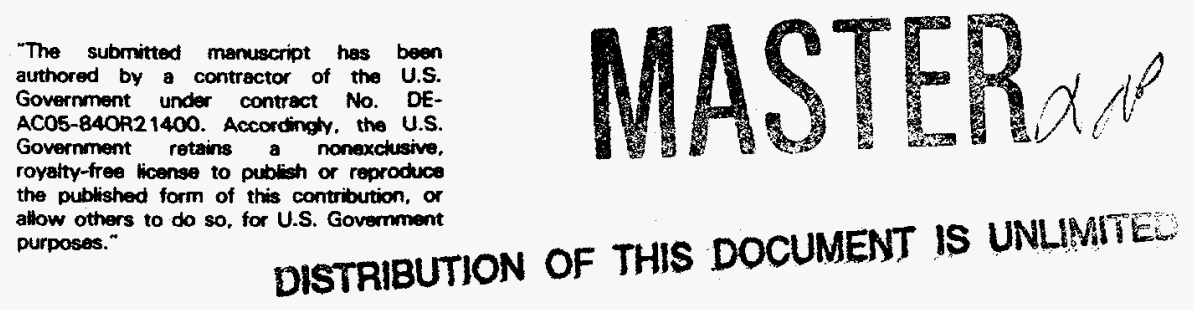
indenter approximates to a parabola of revolution, which is neither sharp at its tip nor properly describes the relationship between depth and contact area, is not evident.

In this paper, results of a finite element simulation are presented which (confirm) the experimentally observed unloading behavior and from which an understanding of the origin of the power law exponents is obtained. The understanding is based on the concept of an effective indenter shape whose geometry is determined by the shape of the plastic hardness impression formed during indentation.

\section{FINITE ELEMENT SIMULATION PROCEDURE}

Elastic/plastic indentation was simulated using the axisymmetric capabilities of the ABAQUS finite element code. As shown in Fig.1, the indenter was modeled as a rigid cone with a half-included angle of $70.3^{\circ}$. This angle gives the same area-to-depth ratio as the Berkovich triangular pyramid used in the nanoindentation experiments of Oliver and Pharr. The specimen was modeled as a large cylinder using the finite element mesh gf'shown in Fig.1. The dark lines along the centerline and the upper surface are a very fine mesh which cannot be resolved in the figure. The fine surface mesh was needed to carefully establish the surface profile.

The material examined in the simulation was aluminum alloy 8009 , which was studied previously by the authors to explore the effects of residual stress on nanoindentation data analysis [4-6]. The elastic/plastic constitutive behavior for the alloy was approximated by piecewise fitting the results of a tension test, as shown in Fig.2, and using the elastic constants $\mathrm{E}=$ $82.12 \mathrm{GPa}$ and $v=0.31$.

\section{RESULTS}

The first finite element simulation was undertaken to establish how well the method works in simulating real experimental behavior. Fig. 3 compares the indentation load-displacement data generated in the simulation to the results of an actual nanoindentation experiment conducted in alloy 8009 . The generally good agreement between the simulation and the experimental curves, both loading and unloading, demonstrates that the finite element method can indeed be

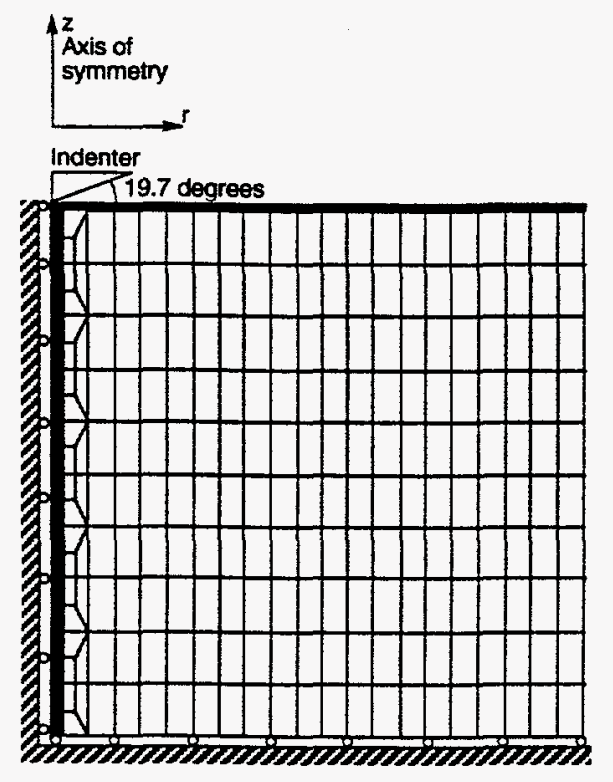

Fig. 1. The mesh and boundary conditions used in finite element simulations.

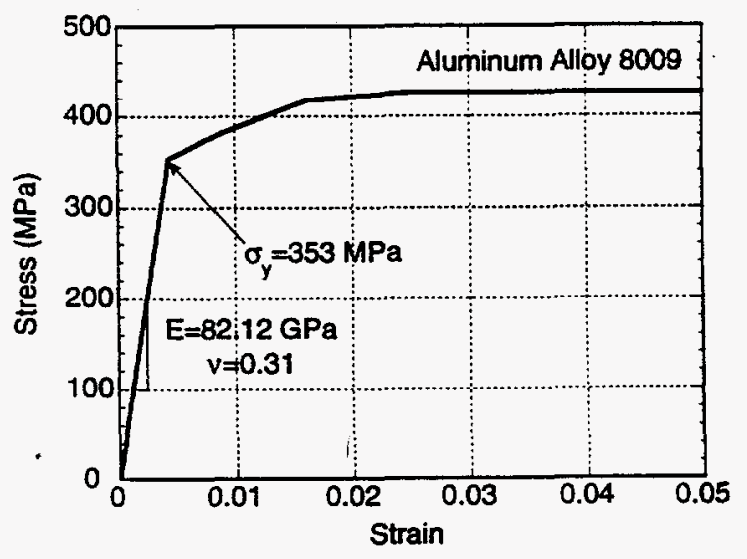

Fig.2. The stress-strain behavior of alloy 8009 used in finite element simulations. 


\section{DISCLAIMER}

Portions of this document may be illegible in electronic image products. Images are produced from the best available original document. 


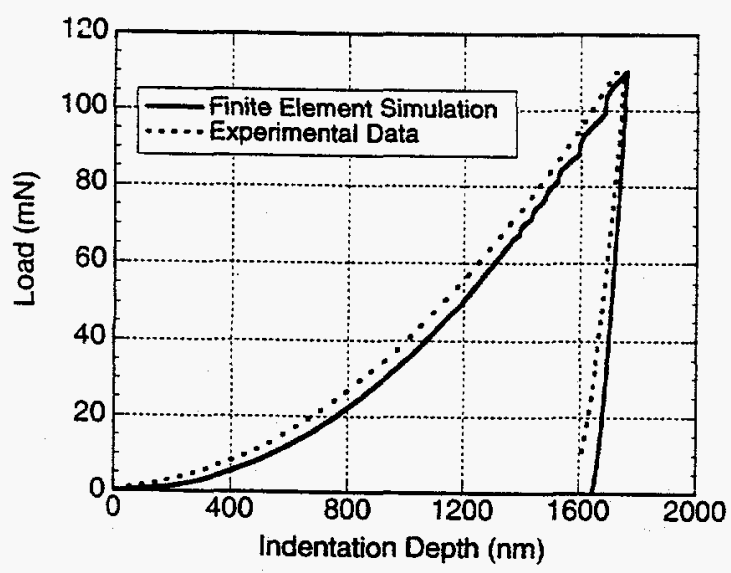

Fig.3. Comparison of indentation loaddisplacement data generated in FE simulation with experimental data.

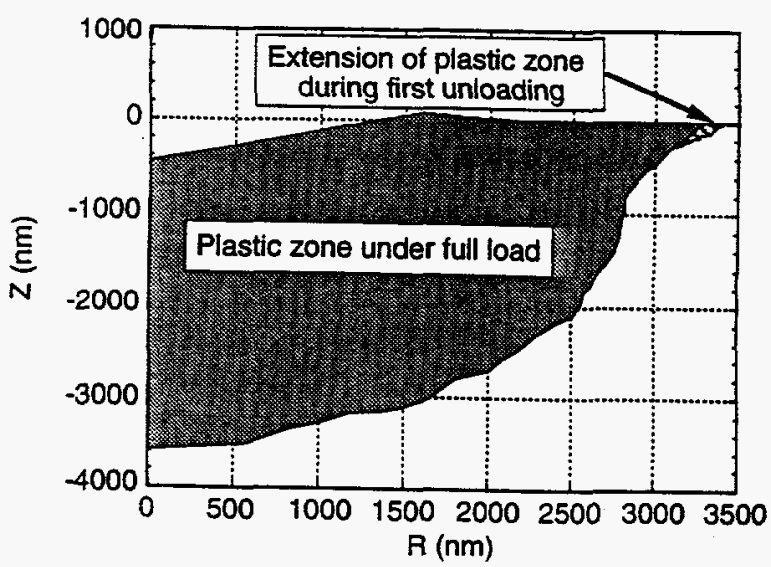

Fig.4. The plastic zone during initial loading and unloading

successfully employed in modeling nanoindentation experiments, and that the $70.3^{\circ}$ cone is a good representation of the Berkovich indenter.

A subsequent simulation was undertaken to explore the extent to which plasticity occurs during unloading and to examine, therefore, how reasonable it is to model the unloading curve from a purely elastic standpoint. In this simulation, the indenter was loaded and unloaded 5 times while plasticity in the vicinity of the indentation and the development of the plastic zone were closely monitored. Fig. 4 shows the plastic zone formed during the initial loading and demonstrates how there is a small increase,the size of the plastic zone near the surface during the first unloading. Thus, some plasticity does indeed occur during unloading, even though the simple constitutive relation used in the simulation does not account for kinematic hardening. In subsequent cycles, further changes in the size and shape of the zone occur, but at progressively smaller rates. However, the unloading plasticity has little influence on the load-displacement behavior. This may be seen in Fig.5, which shows that first and fifth unloading curves as well as the results of a simulation in which the contact impression generated during the first loading cycle was reloaded using an elastic-only constitutive law. The fact that all three curves are virtually indistinguishable indicates that plastic deformation has a negligible influence on the loaddisplacement behavior after the contact impression forms in the first loading. Another significant observation is that all of the unloading curves are well described by the power law of Eqn.1 with a power law exponent $m=1.32$. This exponent, which is in excellent agreement with the value $\mathrm{m}=1.36$ measured in nanoindentation experiments [6], corroborates the experimental findings of Oliver and Pharr ( $\mathrm{m}$ in the range 1.25-1.51) and suggests that there is something fundamental about a power law exponent in this range.

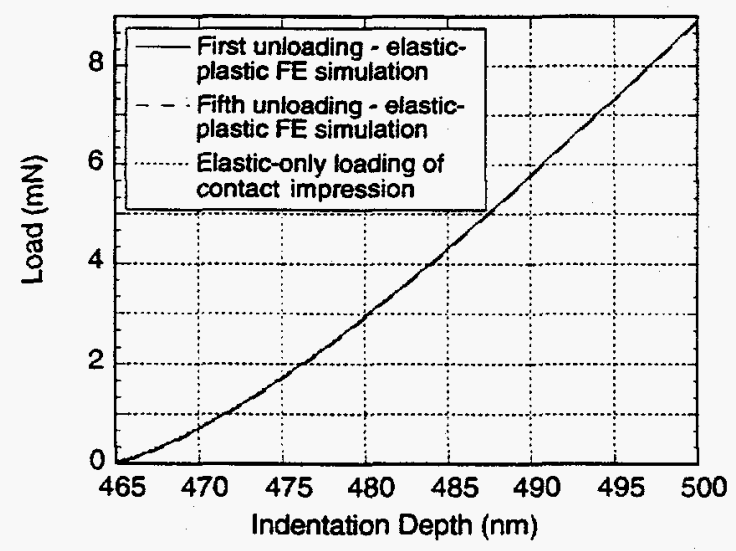

Fig.5. Comparison of the unloading curves obtained in the first and fifth loading cycles with elastic-only loading of the contact impression.

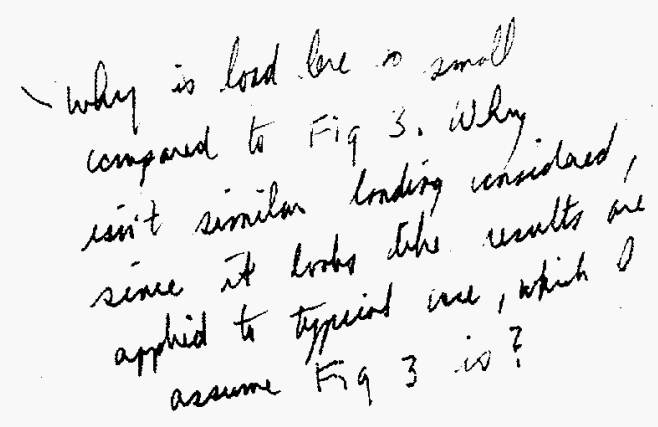


An explanation for why the indentation unloading curves behave as they do is obtained by introducing the concept of an "effective" indenter shape. As illustrated in Fig.6, this concept is used to account for the fact that Sneddon's elastic analysis for a rigid conical punch on a flat surface does not strictly apply for elastic/plastic indentation because of the surface distortions produced during the formation of the permanent hardness impression during the initial loading. What we wish to show, however, is that Sneddon's analysis for the indentation of a flat surface by another axisymmetric indenter can indeed be used to accurately model the load-displacement behavior by defining an "effective" indenter shape. The shape of the "effective" indenter is that which produces the same normal surface displacements on a flat surface that would be produced by the conical indenter on the unloaded, deformed surface of the hardness impression. As such, the shape is described by a function $z=u(r)$, where $u(r)$ is the distance between the conical indenter and the unloaded deformed surface and $r$ is the radial distance from the center of contact (see Fig.6). Thus, provided the shape of the deformed surface is known, the function u( $\hat{\mathbf{z}})$ can be found and the effective indenter can be constructed. From an analytical standpoint, the effective indenter is expected to give a reasonable approximation of the load-displacement behavior for those indenters for which the plastic hardness impression is not greatly displaced from the initial flat surface, i.e., for indenters with relatively large included angles. Finite element simulation is now used to show that for indentation of aluminum alloy 8009 by the $70.3^{\circ}$ cone, the effective indenter does model the simulated load-displacement behavior quite well.

The profile of an unloaded hardness impression made in alloy 8009 by a rigid $70.3^{\circ}$ cone as determined in a finite element simulation was carefully analyzed to establish the function $\mathrm{z}=$ $u(r)$. The particular indentation examined was made to a depth at peak load of $500 \mathrm{~nm}$. At first inspection, the impression appeared to be conical in shape with an apex angle slightly greater than that of the conical indenter. However, closer inspection revealed a subtle curvature in the profile similar to that shown in Fig.6, in which the curvature has been exaggerated for the sake of illustration. From this profile, the shape of the effective indenter was determined and is shown in Fig. 7. The effective indenter is terminated at a radius of $1576 \mathrm{~nm}$, corresponding to the contact edge at full load.

Two features of the effective indenter are worthy of special consideration. First, at the contact edge $(r=1576 \mathrm{~nm})$, the height of the effective indenter is only $12 \mathrm{~nm}$, thus indicating how small the distance is between the conical indenter and the unloaded deformed surface. Second, in sharp contrast to the case of a conical indenter on a flat surface, the effective indenter has a smooth, rounded profile at its tip. The reason for this is that the slope of the deformed hardness impression at its center exactly matches the slope of the conical indenter, i.e., the two surfaces perfectly conform at the tip of the indenter. Thus, the plastic deformation producing the hardness impression has the interesting effect of removing the elastic singularity at the tip of the indenter. the form:

Fig.7 also shows that the effective shape may be approximated by a power law relation of
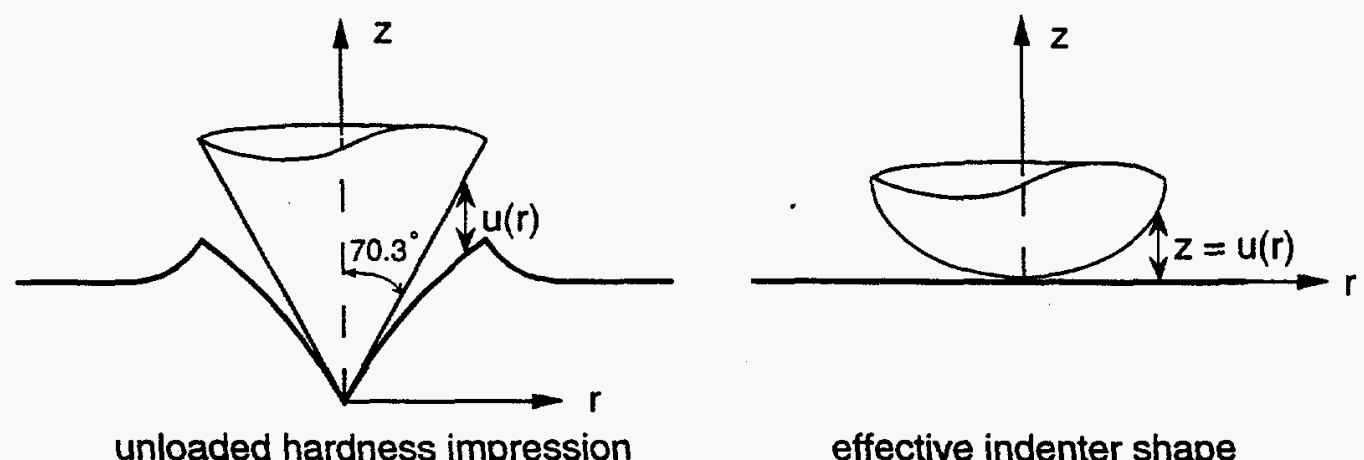

effective indenter shape

Fig.6. The concept of an effective indenter shape. 
where the constants $B$ and $n$ have values $B=4.34 \times 10^{-8} \mathrm{~nm}^{-1.63}$ and $n=2.63$. The effective indenter is thus similar to a parabola of revolution with a slightly higher power law exponent.

That the elastic deformation of a flat surface by the effective indenter closely approximates the elastic deformation of the hardness impression by the conical indenter is shown in Fig.8, which compares load-displacement curves generated in several different ways. The solid line is the first unloading curve determined in the full elastic/plastic finite element analysis for the $70.3^{\circ}$ conical indenter (i.e., the same curve as in Fig. 5). This is to be compared to load-displacement curves for the effective indenter determined in three different ways. The first two were generated by elastic finite element simulation of the deformation of a flat, elastic half-space by the effective indenter; in one, the exact indenter shape was used and in the other, the indenter was constructed from the power law approximation of the indenter shape (Eqn.2). The similarity of these two curves to that for the full elastic/plastic analysis for the conical indenter shows that the effective shape concept does indeed closely approximate the unloading behavior. The third curve for the effective indenter was computed using an analytical approach based on Sneddon's analysis for the deformation of an elastic half-space by a rigid indenter of arbitrary axisymmetric shape [1]. For an indenter described by the general power law of Eqn.2, Sneddon's method yields:

$$
P=\frac{2 E_{e f f}}{(\sqrt{\pi} B)^{1 / n}}\left(\frac{n}{n+1}\right)\left[\frac{\Gamma(n / 2+1 / 2)}{\Gamma(n / 2+1)}\right]^{1 / n} h^{1+1 / n}
$$

where $E_{\text {eff }}=E /\left(1-v^{2}\right)$ is the effective modulus ( $E=$ elastic modulus ; $v=$ Poisson's ratio), $h$ is the elastic displacement, and $\Gamma$ is the factorial (gamma) function. The curve generated from this equation, though slightly lower than those determined in the finite element simulations, is still in reasonably good agreement. The reason that the analytical approach gives a slightly different result is currently under investigation.

One important feature of Eqn.3 is that it directly links the exponent, $n$, describing the effective indenter shape in Eqn.2, to the exponent, $\mathrm{m}$, describing the shape of the unloading curve in Eqn.1; specifically

$$
\mathrm{m}=1+1 / \mathrm{n}
$$

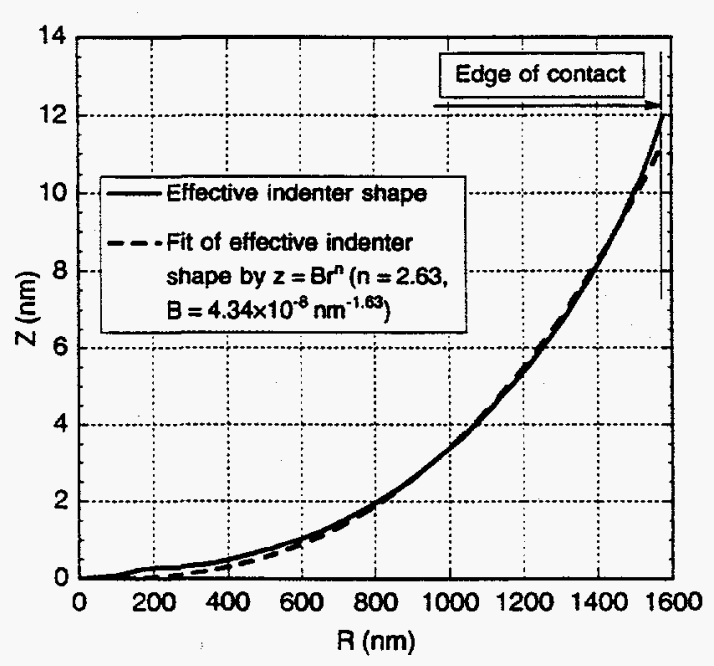

Fig.7. The effective indenter shape and its fit by the power law relation for alloy 8009.

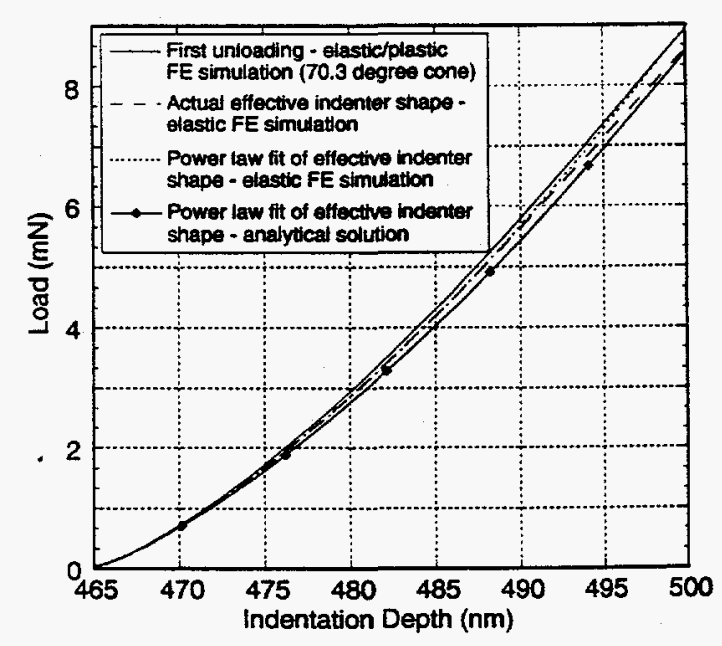

Fig.8. Comparison of load-displacement curves generated in different ways.
$<$

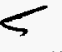

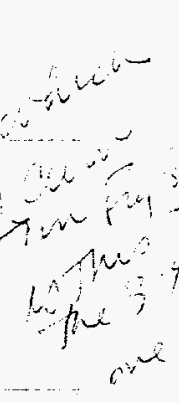

(1) ) 
Since $\mathrm{n}=2.63$ for the effective indenter, Eqn. 4 gives $\mathrm{m}=1.38$ in good agreement with the elastic/plastic finite element simulation and the results of actual nanoindentation experiments.

Overall, these results show that power law unloading behavior with power law exponents in the range $1.3-1.4$ is to be expected in nanoindentation experiments and is explained in terms of the shape of the deformed surface of the hardness impression.

\section{CONCLUSIONS}

1. Finite element simulation of elastic/plastic indentation of an aluminum alloy by a rigid conical indenter with a depth-to-area ratio the same as the Berkovich diamond suggests that indentation unloading curves should be well-described by the power law $\mathrm{P}=\mathrm{A}\left(\mathrm{h}-\mathrm{h}_{\mathrm{f}}\right)^{\mathrm{m}}$ with $\mathrm{m}=1.32$. The value of the exponent is consistent with experimental findings of $\mathrm{m}$ in a variety of materials ranging from 1.25 to 1.51 .

2. Indentation unloading curves may be accurately modeled by the deformation of an eiastic halfspace by an effective indenter whose shape is determined from the profile of the plastic hardness impression. For the aluminum alloy examined in this study, the effective shape is given by $\mathrm{z}=$ $\mathrm{Br}^{2} .63$.

3. While Sneddon's analysis for the deformation of an elastic half-space by a conical indenter predicts $m=2$, the smaller value of $m$ observed in experiments and in finite element simulation is explained by the distortion of the surface from a flat plane configuration caused by the formation of the permanent hardness impression.

\section{ACKNOWLEDGMENTS}

This research was sponsored in part by the Division of Materials Sciences, U.S. Department of Energy, under contract DE-AC05-84OR21400 with Martin Marietta Energy Systems, Inc., and through the SHaRE program under contract DE-AC05-760R00033 with the Oak Ridge Institute for Science and Education.

\section{REFERENCES}

1. I. N. Sneddon, Int. J. Engng. Sci. 3, 47 (1965).

2. M.F. Doerner and W.D. Nix, J. Mater. Res. 1, 601 (1986).

3. W.C. Oliver and G.M. Pharr, J. Mater. Res. 7, 1564 (1992).

4. G.M. Pharr, T.Y. Tsui, A. Bolshakov, and W.C. Oliver, Materials Research Society Symposium Proceedings 338, 127 (1994).

5. A. Bolshakov, W.C. Oliver, and G.M. Pharr, in preparation for submission to J. Mater. Res.

6. T.Y. Tsui, W.C. Oliver, and G.M. Pharr, in preparation for submission to J. Mater. Res.

\section{DISCLAIMER}

\footnotetext{
This report was prepared as an account of work sponsored by an agency of the United States Government. Neither the United States Government nor any agency thereof, nor any of their employees, makes any warranty, express or implied, or assumes any legal liability or responsibility for the accuracy, completeness, or usefulness of any information, apparatus, product, or process disclosed, or represents that its use would not infringe privately owned rights. Reference herein to any specific commercial product, process, or service by trade name, trademark, manufacturer, or otherwise does not necessarily constitute or imply its endorsement, recommendation, or favoring by the United States Government or any agency thereof. The views and opinions of authors expressed herein do not necessarily state or reflect those of the United States Government or any agency thereof.
} 